\title{
Tumour Cells Incorporate Exosomes Derived from Dendritic Cells Through a Mechanism Involving the Tetraspanin CD9
}

\author{
Original Research Article
}

\author{
Graziela Gorete Romagnoli , Patrícia Argenta Toniolo1, Isabela Katz Migliori', \\ Élia Garcia Caldini², Marcelo Alves Ferreira ${ }^{2}$, Célia Regina Pizzo', \\ Patrícia Cruz Bergami-Santos ${ }^{1}$ and José Alexandre M. Barbuto, ${ }^{1, *}$ \\ 1 Department of Immunology, Institute of Biomedical Sciences, University of Sao Paulo, Sao Paulo, SP, Brazil \\ 2 Laboratory of Cell Biology, Faculty of Medicine, University of Sao Paulo, Sao Paulo, SP, Brazil \\ * Corresponding author E-mail: jbarbuto@icb.usp.br \\ Accepted 2 Aug 2012
}

(C) 2013 Romagnoli et al.; licensee InTech. This is an open access article distributed under the terms of the Creative Commons Attribution License (http://creativecommons.org/licenses/by/3.0), which permits unrestricted use, distribution, and reproduction in any medium, provided the original work is properly cited.

Abstract Exosomes (Exos) are secreted nanovesicles that contain membrane proteins and genetic material, which can be transferred between cells and contribute to their communication in the body. We show that Exos, obtained from mature human dendritic cells (DCs), are incorporated by tumour cells, which after Exos treatment, acquire the expression of HLA-class I, HLA-class II, CD86, CD11c, CD54 and CD18. This incorporation reaches its peak eight hours after treatment, can be observed in different cell tumour lines (SK-BR-3, U87 and K562) and could be a means to transform non-immunogenic into immunogenic tumour cells. Interestingly, tetraspanins, which are expressed by the tumour cells, have their surface level decreased after Exo treatment. Furthermore, the intensity of Exo incorporation by the different tumour cell lines was proportional to their CD9 expression levels and pretreatment of Exos with anti-CD9 decreased their incorporation (by SK-BR-3 cells). This modification of tumour cells by DC-derived Exos may allow their use in new immunotherapeutic approaches to cancer. Furthermore, by showing the involvement of CD9 in this incorporation, we provide a possible selection criterion for tumours to be addressed by this strategy.

Keywords Dendritic Cells, Exosomes, Tumour Cells, Immunotherapy, Tetraspanins

\section{Introduction}

Tumour evasion mechanisms to the immune system contribute significantly to the development of neoplasia [1-4]. Among these mechanisms, those affecting tumour antigen presentation, like the effects of the tumour microenvironment upon dendritic cells (DCs) [5], are of major relevance. DCs bridge the innate and adaptive immune responses, being, when properly activated, the only cells able to stimulate naive $\mathrm{T}$ lymphocytes [6-7]. Thus, the inhibition of their maturation within the tumour microenvironment can be seen as a major tumour escape mechanism in its confrontation with the immune system. On the other hand, direct antigen presentation by 
the tumour cells, which lack the ability to deliver the required co-stimulatory signals to $\mathrm{T}$ lymphocytes, may have a similar consequence, that is, the induction of tolerance to the tumour antigens they present [8].

Therefore, immunotherapeutic approaches to cancer that attempt to modify or correct this biased tumour antigen presentation are needed. Many studies explore the potential of in vitro generated DCs to overcome the tumour-induced tolerance, with varying degrees of success [9-13]. Another approach that has been studied is the use of exosomes (Exos) derived from tumour antigenloaded DCs as immunizing agents in experimental models of cancer [14-17]. Exosomes are nanovesicles of $30-100 \mathrm{~nm}$ of diameter that are released from cells as result from the fusion of multivesicular bodies (MVB) with the plasma membrane [18] and which are involved in intercellular communication in the body [19-21]. When originated from DCs, Exos contain many of the molecules involved in antigen presentation, are able to induce direct and indirect lymphocyte responses and can transfer MHC/peptides complex to other DCs [16-17, 22-23].

In this paper we investigated if this transferring of antigen presentation-associated molecules carried by Exos could be observed also when tumour cells are used as "acceptors" of the transfer instead of immune cells. That being the case, DC-derived Exos could be used as a means to turn tolerogenic tumour cells into immunogenic cells, thus contributing to the effectiveness of immunotherapeutic strategies for cancer.

\section{Material and Methods}

\subsection{Sample collection}

Peripheral blood mononuclear cells (PBMCs) were obtained from healthy donor by leukapheresis at the Hospital Alemão Oswaldo Cruz, São Paulo, Brazil. The study was approved by the Institutional Ethics Committee of the ICB-USP (819/CEP). Samples were collected only after informed consent of donors.

\subsection{DCs culture}

Mononuclear cells were separated over a Ficoll-Paque gradient $(\mathrm{d}=1.076)$ (Amersham Pharmacia Biotech, Uppsala, Sweden), resuspended and seeded in six-well plates in serum-free AIM-V ${ }^{\circledR}$ medium $\left(\mathrm{GIBCO}^{\circledR}\right.$, Grand Island, NY, USA). After two hours of incubation at $37^{\circ} \mathrm{C}$, non-adherent cells were removed and the adherent cells (mostly monocytes) were cultured in the presence of GMCSF and IL-4 (50ng/ml; PeproTech, Rocky Hill, NJ, USA) to obtain immature dendritic cells (iDCs). On the fifth culture day, TNF- $\alpha$ (50ng/ml; PeproTech, Rocky Hill, NJ, USA) was added for DC activation. After two further days in culture, mature DCs (mDCs) were harvested and analysed.

\subsection{Cell lines}

The human breast adenocarcinoma cell line SK-BR-3, human leukaemia cell line K562 and human glioblastoma cell line U87 (all free of mycoplasma contamination), were expanded in R10 medium supplemented with $1 \%$ of antibiotic/antimycotic solution $(100 \mathrm{U} / \mathrm{mL}$ penicillin, $100 \mu \mathrm{g} / \mathrm{mL}$ streptomycin and $25 \mu \mathrm{g} / \mathrm{mL}$ amphotericin $\mathrm{B}$, GIBCO $^{\circledR}$, Grand Island, NY, USA).

\subsection{Exosomes purification}

Cell culture supernatants were cleared from cells and submitted to ultracentrifugation for the isolation of nanovesicles. Briefly, supernatant was harvested on day seven of DC cultures and submitted to a series of centrifugations at $4^{\circ} \mathrm{C}: 300 \mathrm{~g}$ (five minutes), $1,200 \mathrm{~g}$ (20 minutes) and 10,000g (30 minutes). After that, the cell and debris-free supernatant was submitted to ultracentrifugation at $100,000 \mathrm{~g}$ for two hours. The pellet, containing nanovesicles enriched in Exos, was resuspended in phosphate-buffered saline (PBS) and ultracentrifuged again at $100,000 \mathrm{~g}$ for two hours. The pellet was resuspended and filter-sterilized $(0.22 \mu \mathrm{m})$. The protein content in this Exo-enriched nanovesicle preparation was assessed by the BCA ${ }^{\mathrm{TM}}$ Protein Assay Kit (Pierce, Rockford, IL, USA). After quantification, the material was aliquoted and stored at $-80^{\circ} \mathrm{C}$. Electron microscopic analysis showed that the pellets contained a nanovesicle-rich material, with particles up to 200nm, which we call, exosomes (Exo).

\subsection{Flow cytometry}

\subsubsection{Monocytes, Dendritic Cells and Tumour Cells}

Cell preparations $\left(2.5 \times 10^{5}\right.$ cells/condition) were labelled with each of the various specific fluorescent antibodies (CD1a, CD9, CD11c, CD14, CD18, CD40, CD54, CD80, CD81, CD83, CD86, CD123 and HLA-DR) (BD Biosciences) and analysed in a FACSCanto II cytometer (Becton Dickinson, San Jose, CA, USA) using the FlowJo software ver. 7.2.4 (Three Star). At least 30,000 events were acquired per antibody analysed.

\subsubsection{Exosomes}

Exo preparations were complexed with paramagnetic beads of $4.5 \mu \mathrm{m}$ (Dynabeads ${ }^{\circledR}$ HLA Cell PrepTM I or II, Invitrogen Dynal AS, Oslo, Norway), similarly to previously described [24]. Exo-bead complexes were incubated in a final volume of $30 \mu \mathrm{L}$, containing $0.5 \%$ albumin Bovine Serum Albumin (BSA) and $3 \mu \mathrm{L}$ of PEconjugated antibody. After 30 minutes of incubation at $4^{\circ} \mathrm{C}$, beads were washed and analysed on a FACS Calibur cytometer (Becton Dickinson, San Jose, CA, USA) using the FlowJo software ver. 7.2.4 (Three Star). 


\subsection{Electron microscopy}

Exo preparations were analysed by transmission electron microscopy. For this, after ultracentrifugation at 100,000g (two hours), the pellet was fixed with $2 \%$ PBSglutaraldehyde and washed with PBS by ultracentrifugation. The pellet was included in araldite resin, cut at a thickness of $80 \mathrm{~nm}$ and post-fixed in osmium by analysis in a JEOL JEM1010 transmission electron microscope.

\subsection{Uptake of Exo by Tumour Cells}

Cells from the SK-BR-3 cell line were incubated at different concentrations and for different amounts of time with Exo at $37^{\circ} \mathrm{C}$ under tension of $5 \% \quad \mathrm{CO}_{2}$. After incubation, tumour cells were treated with the same antibodies used for DCs phenotyping and cell labelling was compared to the non-treated group. Analyses were performed in a FACSCanto II cytometer or in a FACSCalibur cytometer (Becton Dickinson, San Jose, CA, USA).

\subsection{CD9 blocking}

Exo preparations $(130 \mu \mathrm{g} / \mathrm{mL})$ were either pre-incubated or not with purified anti-Human CD9 $(5 \mu \mathrm{g} / \mathrm{mL}$; BDPharmingen) under horizontal agitation for 40 minutes. After this period the material was placed in contact with tumour cells for a period of eight hours. Exoincorporation by tumour cells was determined by labelling of cells with anti-HLA-DR (BD-Pharmingen) followed by flow cytometry.

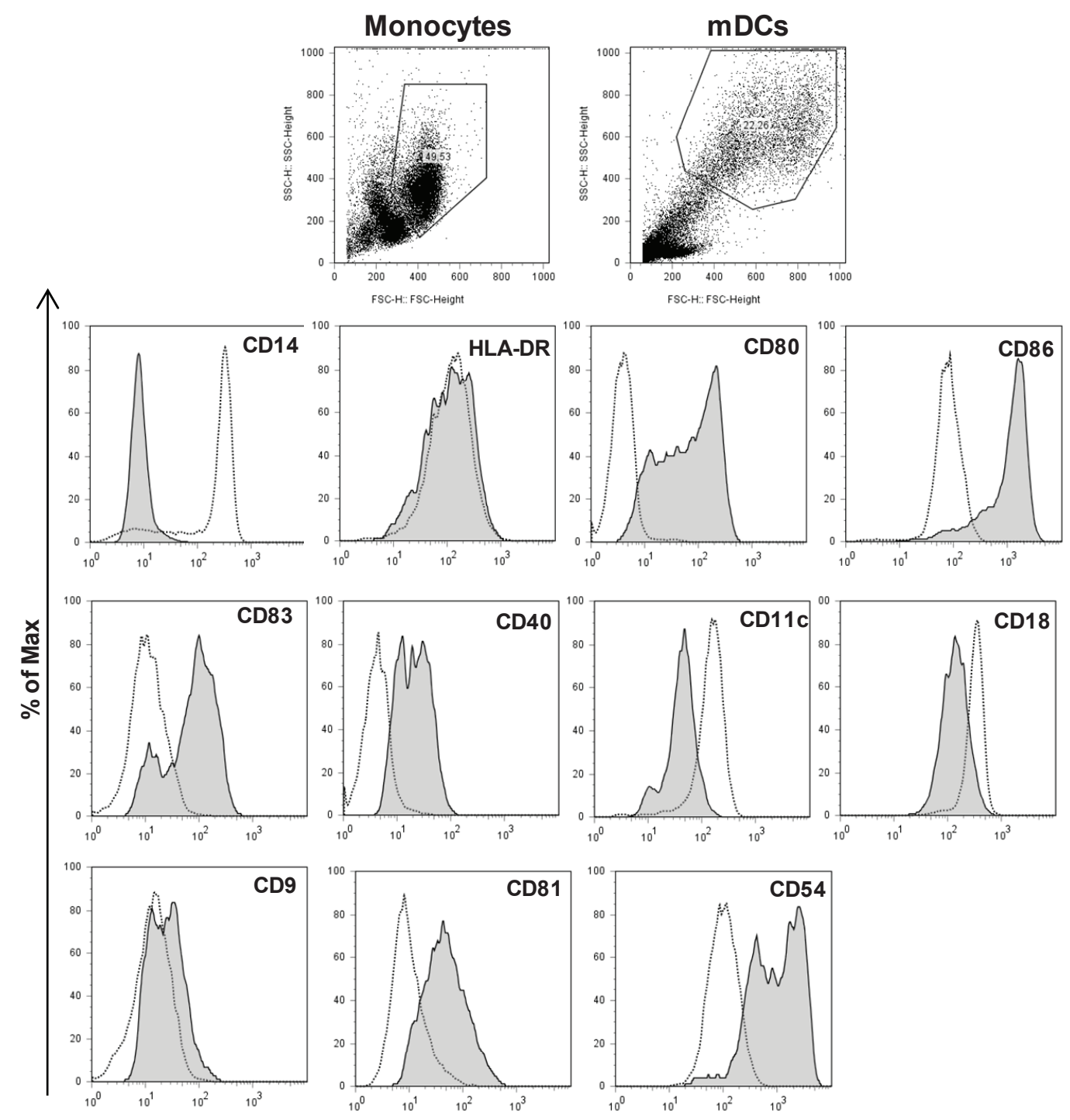

Figure 1. Phenotype of monocytes and mDCs. DCs from healthy donor were generated from monocytes treated with GM-CSF and IL-4 for seven days. At the $5^{\text {th }}$ day of culture TNF- $\alpha$ was added for DC maturation. Dot plots show the analysis gate, based on the size (FSC) and the granularity (SSC) of the cells. Histograms represent the phenotype observed in the monocytes (open histogram) and mDCs (closed histogram). Surface molecules analysed are identified in each histogram. One representative experiment out of eight. 


\subsection{Fluorescence Microscopy}

Exos were labelled with fluorescent wheat germ agglutinin (WGA, Alexa Fluor 633-conjugated, Invitrogen, Carlsbad, CA) and co-incubated for eight hours with tumour SK-BR-3 cells, on glass coverslips in six-well culture dishes. After incubation, the coverslips were thoroughly washed with phosphate-buffered saline and the cells counterstained with DAPI $(10 \mathrm{mg} / \mathrm{mL})$ for 30 minutes at room temperature. After washing, the cells were fixed with $4 \%$ paraformaldehyde for ten minutes at $4{ }^{\circ} \mathrm{C}$, further washed and the coverslips were mounted with glycerol-PBS 1:1 on a microscope slide, sealed with nail polish. The slides were kept in the dark at $4{ }^{\circ} \mathrm{C}$, until reading of a single section on a LSM510 Confocal Zeiss Microscope.

\subsection{Statistical analysis}

Immunophenotyping of exosomes was tested for homogeneity of variances ( $F$ test) followed by $t$ test analysis, if homoscedastic, or the nonparametric Mann-Whitney test, if not. Incorporation assays were tested by a paired $\mathrm{t}$ test and the correlation between CD9 expression and Exo incorporation by different cell lines was analysed by the Pearson's correlation method.

\section{Results}

\subsection{Generation and phenotyping of mature DCs and characterization of exosomes}

mDCs were differentiated from healthy donors' blood monocytes by culture, for seven days, in the presence of GM-CSF, IL-4 and, in the last two days, TNF- $\alpha$. The cells thus generated expressed the expected phenotype of mDCs (Figure 1) with decreased levels of CD14 and increased expression of the co-stimulatory molecules CD80, CD86, CD40. We also analysed the expression of the tetraspanins CD81 and CD9 and the cell adhesion molecule ICAM-1/CD54, which were present and increased in mDCs in comparison to monocytes. On the other hand, the level of the CD18 integrin was slightly decreased in mDCs.

After isolation of Exos by ultracentrifugation of DC culture supernatants, the precipitate was quantified. The protein content originating from $1 \times 10^{6}$ mDCs was $104 \mu \mathrm{g}$ $( \pm 72 \mu \mathrm{g})$. The ultracentrifuged material was analysed by transmission electron microscopy. As we can see in Figure 2, the pellet contained lipid bilayer vesicles of different sizes. Most vesicles had morphology similar to that of endosomal compartment vesicles, with a size range from 30 to $200 \mathrm{~nm}$. This includes the expected size for exosomes (30-100nm).


Figure 2. Electron micrograph of nanovesicles obtained in the supernatant of mDCs. The pellet obtained by ultracentrifugation of supernatant from mature DCs was processed and analysed by transmission electron microscopy. The vesicles have a size range 30 to 200nm. (A) 24,000X, scale bar, 1 $\mu \mathrm{m}$; (B) 39,000X, scale bar, 500nm; (C) and (D) 93,000X, scale bar, 200nm. 


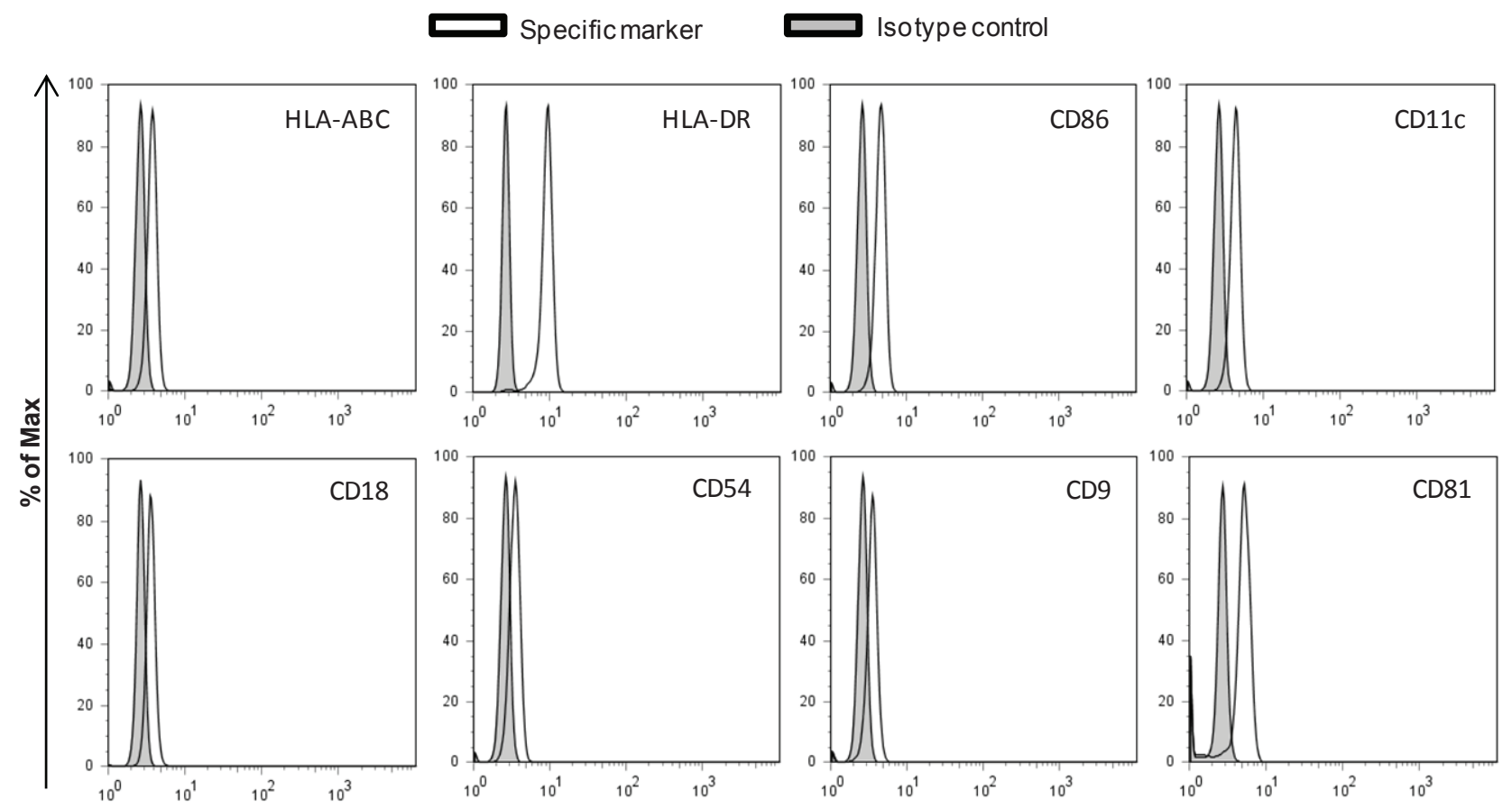

Figure 3. Presence of molecules involved in lymphocyte activation and cell adhesion in Exos from mDCs. Exos were coupled with paramagnetic beads labelled with anti-MHC-I or anti-MHCII, as described in Materials and Methods. After coupling, Exos were incubated for 30 minutes $\left(4^{\circ} \mathrm{C}\right.$ in the dark) with monoclonal antibodies for the specific molecules. Representative histograms of surface markers found in Exos, where open histograms represent relevant Ab-labelled beads and closed histogram represent fluorescence of beads stained with isotype controls. Surface molecules analysed are identified in each histogram.

We also analysed the precipitated material by flow cytometry. Exo from mDCs $(n=9)$ consistently carried the molecules involved in antigen presentation and lymphocyte activation, HLA-ABC $(\mathrm{p}=0.02)$ and CD86 $(p=0.005)$ and, in some preparations, also HLA-DR. Exos also presented molecules involved in cell adhesion, such as integrins and tetraspanins CD11c $(\mathrm{p}=0.008), \mathrm{CD} 18$ $(p=0.007), \operatorname{CD} 81(p=0,003)$ and CD9 $(p=0.03)$.

\subsection{Exo obtained from mDCs change the phenotype of tumour cells}

It is known that DCs are able to capture Exos originating from other DCs and present molecules from these nanovesicles on their surface. Here, we decided to analyse if Exos that originated from mDCs would be incorporated by tumour cells and cause a similar phenotypic modification. To evaluate this, we stained $\mathrm{mDC}$-originated Exo with fluorescent-wheat germ agglutinin (WGA) and treated cells of three different human tumour cell lines (the SK-BR-3 breast adenocarcinoma, the K562 leukaemia and the U87 glioblastoma) for eight hours with WGA-labelled Exos or with non-labelled Exos. After this period, the incorporation of WGA-labelled Exos by the tumour cells was determined by flow cytometry (Figure 4). As shown, cells from the three cell lines incorporated WGA-labelled Exos. This incorporation was also confirmed by fluorescence microscopy (Figure 5).
Finally, we determined if antigen presentation-associated molecules, present in the Exos would be incorporated by tumour cells and what the kinetics would be in this incorporation. For this, we treated $1 \times 10^{6}$ cells of the human breast adenocarcinoma cell line, SK-BR-3, with $65 \mu \mathrm{g}$ of Exos for different times. Exo-treatment caused time-depended tumour cell-labelling for HLA-DR and CD86, both molecules that were absent from untreated cells (Figure 6).

Interestingly, when we evaluated the levels of the tetraspanins, CD9 and CD81 on Exo-treated SK-BR-3 cells, we noticed that the levels of these molecules were decreased in comparison to untreated cells (significantly for CD9 and marginally significant for CD81), a phenomenon that was not noticed in the expression of CD54, another molecule also expressed by the untreated tumour cells (Figure 7A and B). This decrease was consistently observed in different experiments, as shown in Figure 7C. Furthermore, we noted that the intensity of Exo incorporation by the three different tumour cell lines was proportional to the levels of CD9 expression by these (Figure 7D).

\subsection{Involvement of CD9 tetraspanin in incorporation of Exo by tumour cells}

Tetraspanins are involved with cellular adhesion and the level of Exo incorporation by tumour cells was 
proportional to the level of CD9 expressed by them. In addition, CD9 and CD81 expression were decreased after Exo-treatment of the tumour cells. These data suggested that these molecules were involved also in the incorporation of Exo by the tumour cells. In order to confirm the participation of CD9 in the phenomenon, we blocked CD9 during the incubation of Exo with tumour cells and evaluated its effects upon the incorporation of Exo by the tumour cells. For this, $1 \times 10^{6}$ tumour cells were treated with $130 \mu \mathrm{g}$ of Exo from mDCs, which were pre- incubated or not with purified anti-CD9 $(5 \mu \mathrm{g} / \mathrm{mL})$. After the incubation, tumour cells were labelled with anti-HLADR to evaluate Exo incorporation. As shown in Figure 8, addition of CD9 to Exos did not completely inhibit the incorporation of HLA-DR by the tumour cells, but resulted in a decrease in the percentage of labelled cells (from 35\% to $21 \%$ ) and in the intensity of labelling (from 547 to 350 in MFI). The treatment with isotype antibody controls also caused a decrease in Exo incorporation, but this was not as significant (data not shown).
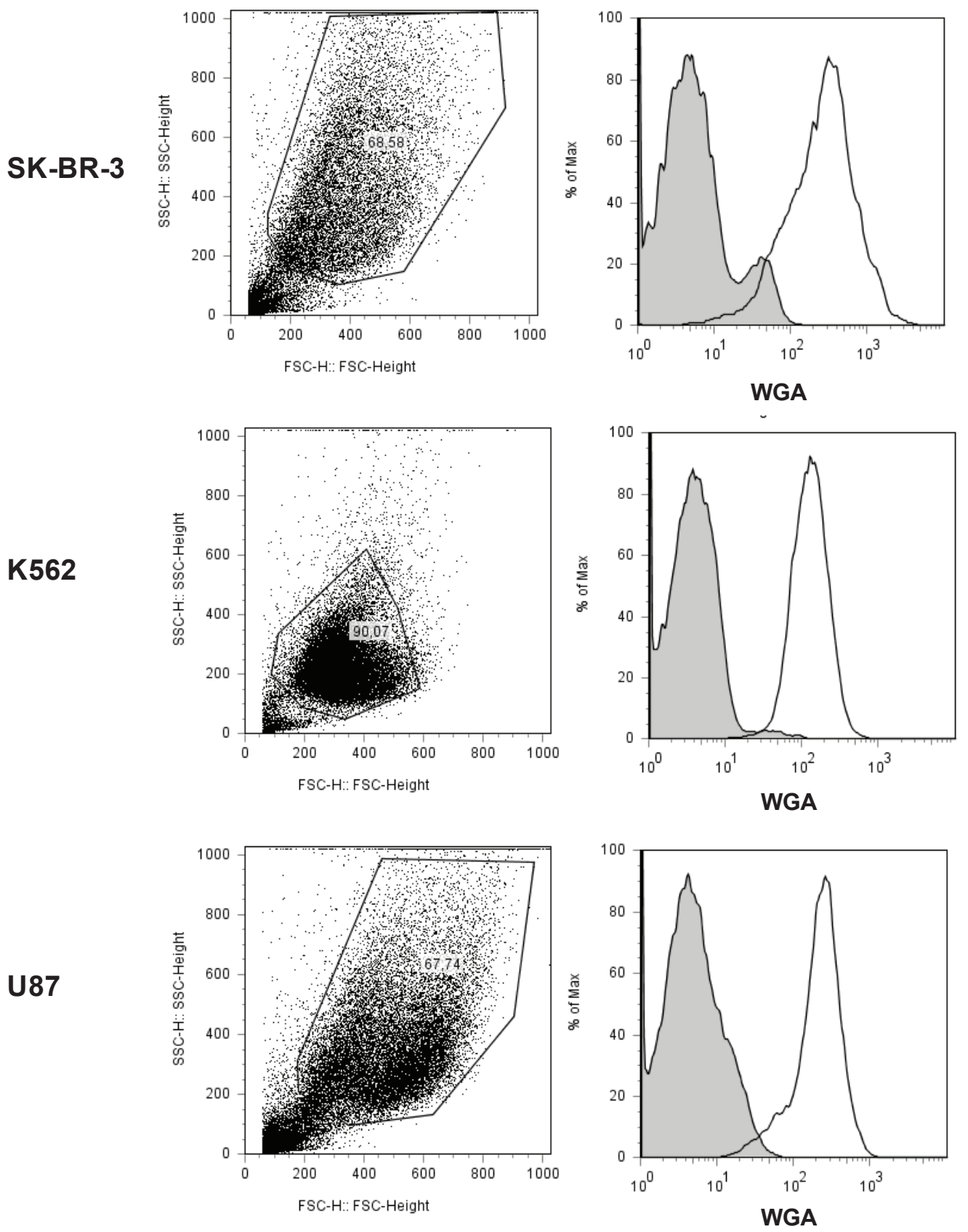

Figure 4. Incorporation of Exo by SK-BR-3, K562 and U87 cell lines. Exo from DCs were stained with WGA (Wheat Germ Agglutinin Conjugates - InvitrogenTM (Alexa Fluor 633). After this, tumour cells were treated for eight hours with Exos or Exo/WGA. After the incubation, the cells were evaluated by flow cytometry. On the left, Dot plots showing the main gate analysis based on the size (FSC) and the granularity (SSC), for each tumour cell line; on the right, histograms showing the Exo/WGA incorporation; open histograms represent tumour cells treated with Exos stained with WGA and the closed histograms represent tumour cells treated with non-labelled Exos. 

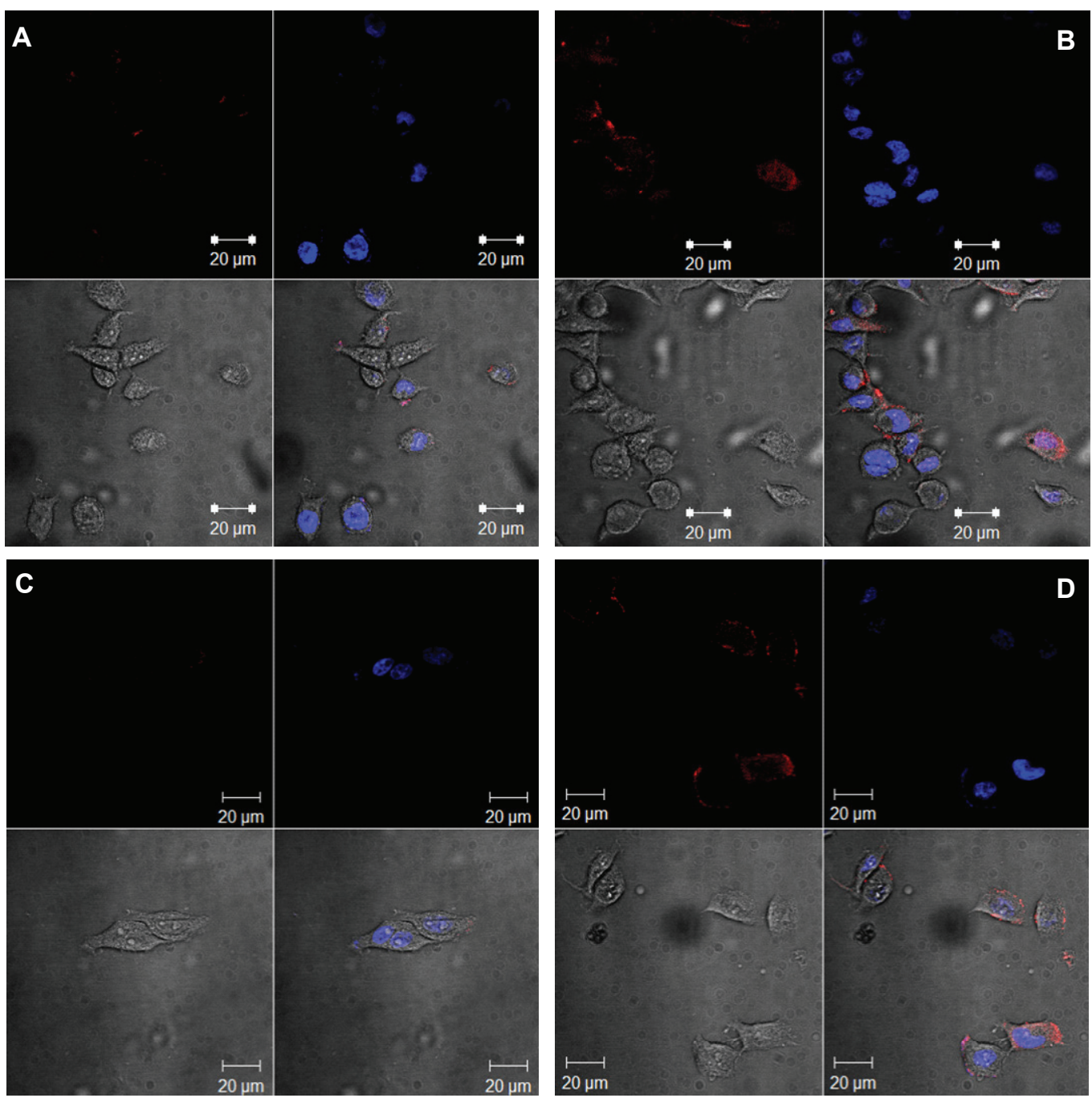

Figure 5. Incorporation of Exo by tumour cells of the human breast adenocarcinoma cell line, SK-BR-3. Tumour cells were treated for 8 hours with Exo from mDCs, which were previously labelled with Wheat Germ Agglutinin Conjugates - InvitrogenTM (Alexa Fluor 633). After incubation, the cells were processed for analysis by fluorescence microscopy. (A) and (C) represent two fields of control staining with DAPI of cells treated with non-labelled Exos. (B) and (D) represent two fields of cells treated with red fluorescence labelled Exo. Objective 63X in oil immersion.

\section{Discussion}

Our data show that exosomes from mature DCs are able to modify the phenotype of tumour cells, which, after exposure to these nanovesicles, present at their membrane, Exo-carried molecules associated with antigen presentation. Exos are nanovesicles of endosomal source that contribute to the membrane traffic between different cell types [20,25]. In the immune system Exos were shown to transfer information and function from the cell that produced the Exos to those incorporating them [21-22]. Therefore, the actual incorporation of Exos from DCs by tumour cells, as shown here, opens new possibilities for immunotherapy, based on the use of such Exo-treated tumour cells as antigen-presenting cells of their own tumour antigens.
Nanovesicles were obtained from DCs that were differentiated from blood monocytes obtained from healthy donors by the usual methods [26]. After DC activation, supernatants were collected, cleared from cells and submitted to ultracentrifugation for the isolation of nanovesicles. The products of these preparations were examined by transmission electron microscopy and showed, in fact, the presence of vesicles of 30 to $200 \mathrm{~nm}$, with lipid bilayer membranes and morphology is compatible with an endosomal origin. Contamination of our preparations by apoptotic bodies and shedding microvesicles cannot be excluded, but their frequency should not be high. Cell viability, which was above $90 \%$, the lack of highly electrondense material, a characteristic of apoptotic bodies [27] and the absence of CD40, which has been shown to be characteristic of shedding microvesicles [28], suggest that these types of vesicles 
were not predominant in our preparations. On the other hand, Exos have been described as vesicles with less than $100 \mathrm{~nm}$ [28] and though larger particles were also present in our preparations, the flow cytometry data, demonstrating the presence of molecules typically described in Exos derived from DCs, such as HLA-ABC, HLA-DR, CD86, CD11c, CD54, CD18 and also CD81 and CD9, tetraspanins hallmarks of Exo [14, 17, 24; 27, 29-30], together with the morphology of the vesicles lead us to consider the preparations as Exo preparations.
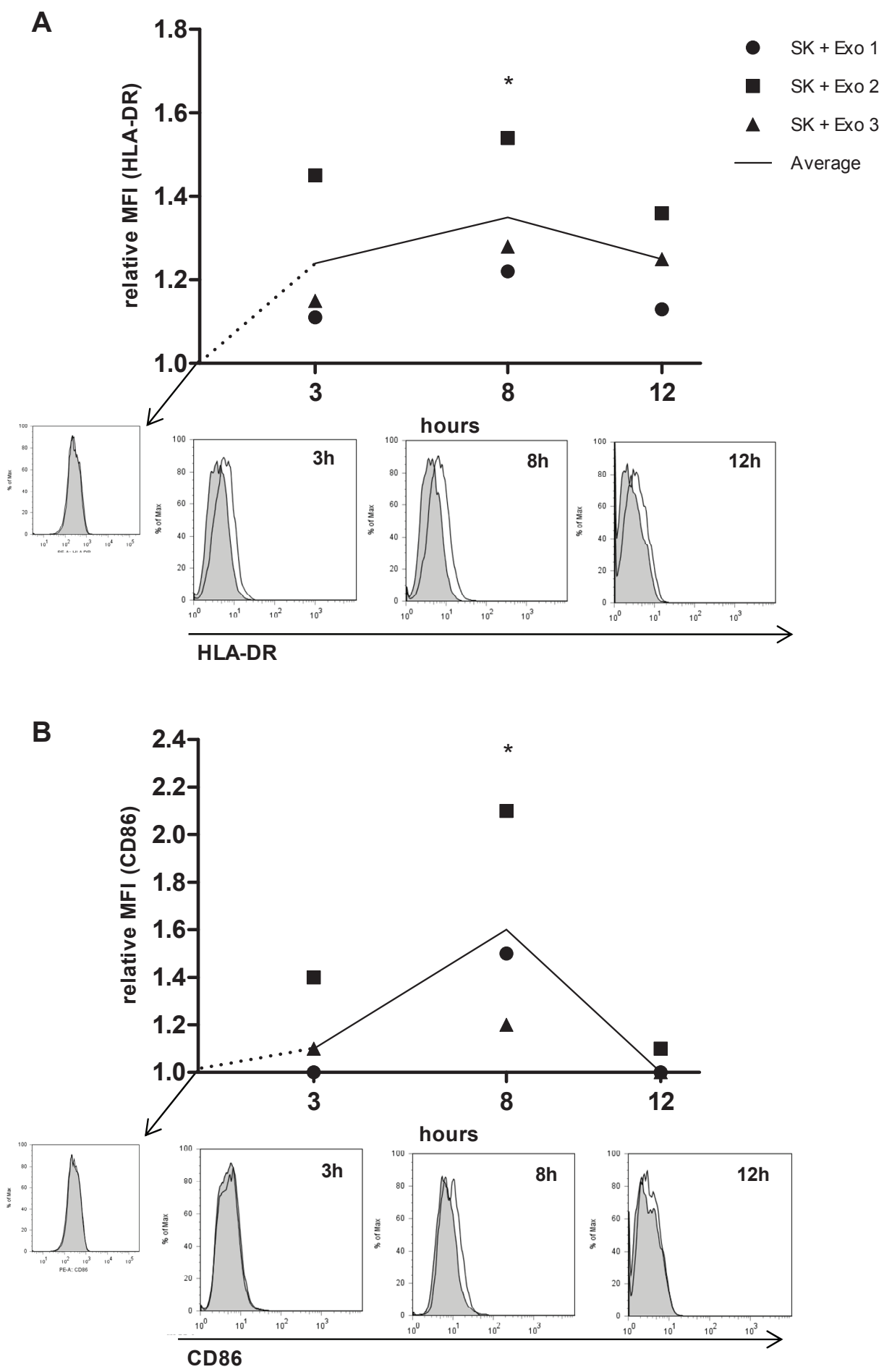

Figure 6. Kinetics of HLA-DR and CD86 incorporation by SK-BR-3 cells after treatment with DC-originated Exos, where Exo-1, Exo-2 and Exo-3 represent three different preparations. Relative increases in MFI for the molecules (A) HLA-DR and (B) CD86 are shown in the upper graphs in (A) and (B), while the histograms below show the basal level of expression of the marker by untreated cells (grey histograms) and treated cells (empty histograms) of a representative experiment out of three; ${ }^{*}$ at eight hours, $\mathrm{n}$ was increased to six and, for this time point, the differences in expression of HLA-DR and CD86, by Exo-treated and non-treated cells had a $p=0.02$. 

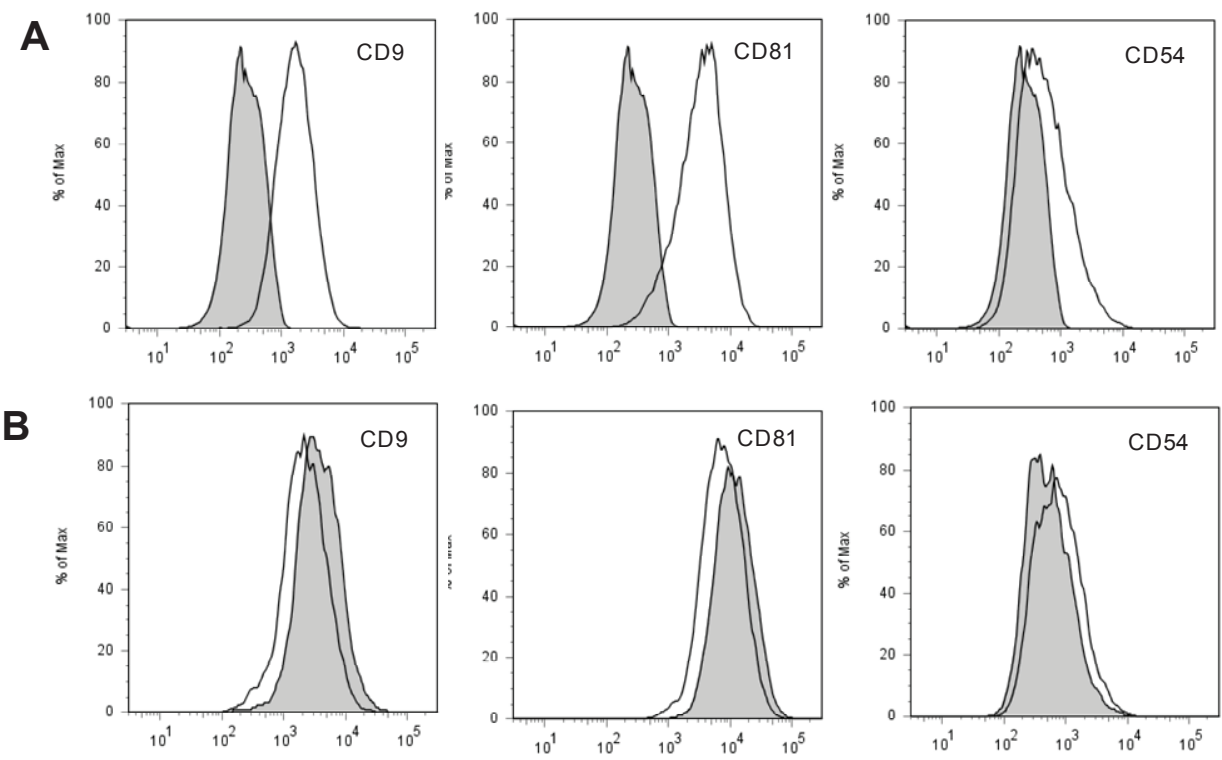

C

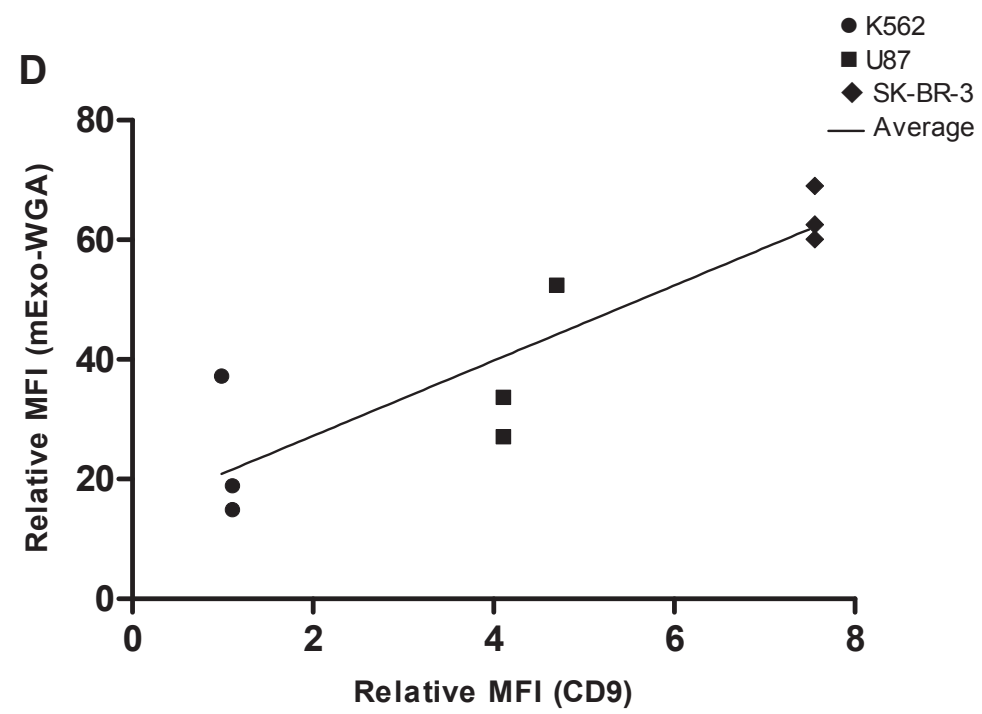

Figure 7. Effect of Exo treatment upon surface molecules of tumour cells. (A) Surface phenotype of SK-BR-3 cells (open histograms represent cells labelled with specific antibodies and closed histograms represent cells stained with isotype controls). (B) Representative histograms showing the phenotype of Exo-treated tumour cells after eight hours of incubation. (C) Relative Median Fluorescence Intensities (MFI) of the molecules analysed before and after treatment of tumour cells with different preparations of nanovesicles. The relative MFI was obtaining by the division of the MFI of the treated group by the MFI of the untreated group in the same experiment. (D) Relationship between CD9 expression by the cells from the different lines (expressed as relative MFI - in comparison to non-labelled cells) and Exo incorporation by the cells. (Pearson' $r=0.89 ; \mathrm{p}=0.001$ ).

In agreement with what has been described for other cell types [20, 22, 25, 31], tumour SK-BR-3 cells also acquired Exo-carried molecules, as detected by flow cytometry and confirmed by fluorescence microscopy. The kinetics of this incorporation was similar in repeated experiments and indicated that the peak expression of Exo-carried molecules was achieved eight hours after exposure of the tumour cells to the Exos. Intriguingly however, the levels of the tetraspanins CD9 and CD81, which were molecules present in the tumour cell line and in the Exos, did not increase, but decreased on the surface of Exo-treated tumour cells.
Actually, CD9, CD81, MHC-class I, T-cell receptors, CD54 and LFA-1 have been shown to affect the uptake of Exos by different cell types [22, 25, 32-34]. Furthermore, mechanisms involved in this incorporation may vary among cell types and activation status both of target and source cells. Endothelial cells incorporate Exo from DCs with peaks between three and six hours through a receptor-mediated endocytosis, showing a decrease in Exo-derived molecules after 24 hours and dependence on the activation status of the DCs [35], immature DCs incorporate Exo better than mature DCs [34] and annexins and lipid rafts affect Exo incorporation by tumour cells [36], as well as their adhesion and spreading. 




B

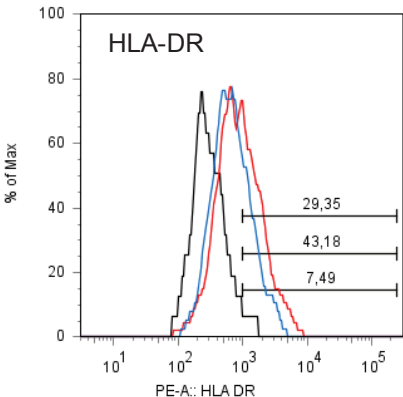

\begin{tabular}{|c|c|c|}
\hline & SK + Exo & SK + (Exo/anti-CD9) \\
\hline HLA-DR $^{+}$cells (\%) & 35 & 21 \\
\hline MFI & 547 & 350 \\
\hline
\end{tabular}

Figure 8. Involvement of CD9 tetraspanin in Exo incorporation by tumour cells. Exos (130 $\mu \mathrm{g})$ were pre-treated or not with anti-CD9 for 40 minutes. After that, Exos were incubated for eight hours with SK-BR-3 tumour cells and these were analysed by flow cytometry. (A) Histograms showing the Median Fluorescence Intensity (MFI) of the HLA-DR marker in the various groups (black line, isotype control and red line, specific marker); (B) Overlay of HLA-DR MFI: the bars represent the percentages of positive cells, the black line represents the untreated group, the red line represents the group treated with Exo without anti-CD9 and the blue line represents the group treated with Exos and pre-incubated with anti-CD9. The table summarizes the results.

Our data show that the incorporation of Exos by different tumour cell lines (SK-BR-3, K562 and U87) was proportional to the level of CD9 expressed by the cells before treatment, indicating that this tetraspanin may have a significant role in this phenomenon. Tetraspanins, like CD9 and CD81, don't have a specific receptor, but, rather, show a promiscuous association with other proteins, such as integrins [37], other tetraspanins [38-39] and MHC class I and II molecules [30, 40]. These molecules constitute a hallmark of exosomes [30] and are believed to facilitate the incorporation of proteins into MVB by their ability to form aggregates with them [41]. Despite the role of tetraspanins in protein association and incorporation by other cell types, their role in Exo incorporation by tumour cells had not been described until now. However, the facts that CD9 levels decreased in Exotreated cells and that the level of Exo incorporation by different cell lines was proportional to their CD9 expression pointed to a relevant role for this molecule in the interaction between Exos and the tumour cells. Indeed, in agreement with this role, we noted that the treatment of Exos with anti-CD9 decreased their incorporation by the tumour cells. It must be considered, however, that the treatment of Exo with control antibodies also caused a decrease in Exo incorporation by the cells, as shown in the literature [32], though not as intense.

Although we did not test directly, CD81, another typical tetraspanin of Exos [20, 40] may also to be involved in their incorporation by tumour cells, since, as those of
CD9, its levels were decreased in Exo-treated cells (though not as significantly). Furthermore, K562 cells, which did not express CD9, incorporated Exos, demonstrating that even if CD9 is involved in the Exo incorporation by the cells, it is not the only molecule involved in the phenomenon.

In conclusion, the data presented here show that exosomes derived from mature dendritic cells are incorporated by different tumour cell lines, transferring to these cells a series of surface molecules associated with antigen presentation and the consequences of this incorporation are currently under investigation. By itself, this observation can provide support to the development of new immunotherapeutic strategies against cancer, exploiting the modification of tumour cells into potentially more immunogenic cells. Furthermore, the data indicate a possible role for the tetraspanins CD9 in the incorporation of Exos by tumour cells, thus providing a possible selection criterion for the choice of tumours to be addressed by this strategy: the level of CD9 expression by the tumour cells.

\section{Acknowledgements}

This study was supported by grants (\#04/09956-0; \#07/58597-1; \#09/54599-5) from the Fundação de Amparo a Pesquisa do Estado de São Paulo (FAPESP) and the Conselho Nacional de Desenvolvimento Científico e Tecnológico, CNPq (\#303731/2007-9). 


\section{References}

[1] Almand B, Resser JR, Lindman B, Nadaf S, Clark JI, Kwon ED, Carbone DP, Gabrilovich DI (2000) Clinical significance of defective dendritic cell differentiation in cancer. Clin. Cancer Res. 6(5): 1755-66.

[2] Bodmer WF, Browning MJ, Krausa P, Rowan A, Bicknell DC, Bodmer JG (1993) Tumor escape from immune response by variation in HLA expression and other mechanisms. Ann. N. Y. Acad. Sci. 690: 42-9.

[3] Tada T, Ohzeki S, Utsumi K, Takiuchi H, Muramatsu M, Li XF, Shimizu J, Fujiwara H, Hamaoka T (1991) Transforming growth factor-beta-induced inhibition of $\mathrm{T}$ cell function. Susceptibility difference in $\mathrm{T}$ cells of various phenotypes and functions and its relevance to immunosuppression in the tumorbearing state. J. Immunol. 146(3): 1077-82.

[4] Fiorentino DF, Zlotnik A, Vieira P, Mosmann TR, Howard M, Moore KW, O'Garra A (1991) IL-10 acts on the antigen-presenting cell to inhibit cytokine production by Th1 cells. J. Immunol. 146(10): 3444-51.

[5] Baleeiro RB, Anselmo LB, Soares FA, Pinto CA, Ramos O, Gross JL, Haddad F, Younes RN, Tomiyoshi MY, Bergami-Santos PC, Barbuto JA (2008). frequency of immature dendritic cells and altered in situ production of interleukin-4 and tumor necrosis factor-alpha in lung cancer. Cancer Immunol. Immunother. 57(9):1335-45.

[6] Banchereau J, Steinman RM (1998) Dendritic cells and the control of immunity. Nature. 392(6673): 245-52.

[7] Steinman RM (1991) The dendritic cell system and its role in immunogenicity. Annu Rev. Immunol. 9: 27196.

[8] Drake CG, Jaffee E, Pardoll DM (2006) Mechanisms of immune evasion by tumors. Adv. Immunol. 90: 51-81.

[9] Barbuto JA, Ensina LF, Neves AR, Bergami-Santos P, Leite KR, Marques R, Costa F, Martins SC, CamaraLopes LH, Buzaid AC (2004) Dendritic cell-tumor cell hybrid vaccination for metastatic cancer. Cancer Immunol. Immunother. 53(12): 1111-8.

[10] Gong J, Nikrui N, Chen D, Koido S, Wu Z, Tanaka Y, Cannistra S, Avigan D, Kufe D (2000) Fusions of human ovarian carcinoma cells with autologous or allogeneic dendritic cells induce antitumor immunity. J. Immunol. 165(3): 1705-11.

[11] Kugler A, Stuhler G, Walden P, Zöller G, Zobywalski A, Brossart P, Trefzer U, Ullrich S, Müller CA, Becker V, Gross AJ, Hemmerlein B, Kanz L, Müller GA, Ringert RH (2000) Regression of human metastatic renal cell carcinoma after vaccination with tumor cell-dendritic cell hybrids. Nat. Med. 6(3): 332-6.

[12] Nestle FO, Alijagic S, Gilliet M, Sun Y, Grabbe S, Dummer R, Burg G, Schadendorf D (1998) Vaccination of melanoma patients with peptide- or tumor lysate-pulsed dendritic cells. Nat. Med. $4(3): 328-32$.
[13] Murphy G, Tjoa B, Ragde H, Kenny G, Boynton A (1996) Phase I clinical trial: T-cell therapy for prostate cancer using autologous dendritic cells pulsed with HLA-A0201-specific peptides from prostate-specific membrane antigen. Prostate. 29(6): 371-80.

[14] Viaud S, Ploix S, Lapierre V, Théry C, Commere PH, Tramalloni D, Gorrichon K, Virault-Rocroy P, Tursz T, Lantz O, Zitvogel L, Chaput N (2011) Updated technology to produce highly immunogenic dendritic cell-derived exosomes of clinical grade: a critical role of interferon- $\gamma$. J. Immunother. 34(1): 65-75.

[15] Viaud S, Théry C, Ploix S, Tursz T, Lapierre V, Lantz O, Zitvogel L, Chaput N (2010) Dendritic cell-derived exosomes for cancer immunotherapy: what's next? Cancer Res. 70(4): 1281-5

[16] Chaput N, Schartz NE, André F, Taïeb J, Novault S, Bonnaventure P, Aubert N, Bernard J, Lemonnier F, Merad M, Adema G, Adams M, Ferrantini M, Carpentier AF, Escudier B, Tursz T, Angevin E, Zitvogel L. Exosomes as potent cell-free peptidebased vaccine. II (2004) Exosomes in CpG adjuvants efficiently prime naive Tc1 lymphocytes leading to tumor rejection. J. Immunol. 172(4): 2137-46.

[17] Zitvogel L, Regnault A, Lozier A, Wolfers J, Flament C, Tenza D, Ricciardi-Castagnoli P, Raposo G, Amigorena S (1998) Eradication of established murine tumors using a novel cell-free vaccine: dendritic cellderived exosomes. Nat. Med. 4(5): 594-600.

[18] Raposo G, Nijman HW, Stoorvogel W, Liejendekker R, Harding CV, Melief CJ, Geuze HJ (1996) B lymphocytes secrete antigen-presenting vesicles. J. Exp. Med. 183(3): 1161-72.

[19] Camussi G, Deregibus MC, Bruno S, Grange C, Fonsato V, Tetta C (2011) Exosome/microvesiclemediated epigenetic reprogramming of cells. Am. J. Cancer Res. 1(1):98-110.

[20] Mathivanan S, Ji H, Simpson RJ (2010) Exosomes: extracellular organelles important in intercellular communication. J. Proteomics. 2010. 73(10): 1907-20.

[21] Denzer K, van Eijk M, Kleijmeer MJ, Jakobson E, de Groot C, Geuze HJ (2000) Follicular dendritic cells carry MHC class II-expressing microvesicles at their surface. J. Immunol. 165(3): 1259-65.

[22] Hao S, Bai O, Li F, Yuan J, Laferte S, Xiang J (2007) Mature dendritic cells pulsed with exosomes stimulate efficient cytotoxic T-lymphocyte responses and antitumour immunity. Immunology. 120(1): 90-102.

[23] Hao S, Bai O, Yuan J, Qureshi M, Xiang J (2006) Dendritic cell-derived exosomes stimulate stronger CD8+ CTL responses and antitumor immunity than tumor cellderived exosomes. Cell Mol. Immunol. 3(3): 205-11.

[24] Clayton A, Court J, Navabi H, Adams M, Mason MD, Hobot JA, Newman GR, Jasani B (2001) Analysis of antigen presenting cell derived exosomes, based on immuno-magnetic isolation and flow cytometry. J. Immunol. Methods. 247(1-2):163-74. 
[25] Nolte-'t Hoen EN, Buschow SI, Anderton SM, Stoorvogel W, Wauben MH (2009) Activated T cells recruit exosomes secreted by dendritic cells via LFA1. Blood. 113(9): 1977-81.

[26] Sallusto F, Lanzavecchia A (1994) Efficient presentation of soluble antigen by cultured human dendritic cells is maintained by granulocyte/macrophage colony-stimulating factor plus interleukin 4 and downregulated by tumor necrosis factor alpha. J. Exp. Med. 179(4): 1109-18.

[27] Théry C, Boussac M, Véron P, Ricciardi-Castagnoli $P$, Raposo G, Garin J, Amigorena S (2001) Proteomic analysis of dendritic cell-derived exosomes: a secreted subcellular compartment distinct from apoptotic vesicles. J. Immunol. 166(12): 7309-18.

[28] Mathivanan S, Simpson RJ (2009) ExoCarta: A compendium of exosomal proteins and RNA. Proteomics. 9(21):4997-5000.

[29] Segura E, Amigorena S, Théry C (2005) Mature dendritic cells secrete exosomes with strong ability to induce antigen-specific effector immune responses. Blood Cells Mol. Dis. 35(2): 89-93.

[30] Théry C, Zitvogel L, Amigorena S (2002) Exosomes: composition, biogenesis and function. Nat. Rev. Immunol. 2(8): 569-79.

[31] Davis DM (2007) Intercellular transfer of cellsurface proteins is common and can affect many stages of an immune response. Nat. Rev. Immunol. 7(3): 238-43.

[32] Xie Y, Zhang H, Li W, Deng Y, Munegowda MA, Chibbar R, Qureshi M, Xiang J (2010) Dendritic cells recruit $\mathrm{T}$ cell exosomes via exosomal LFA-1 leading to inhibition of CD8+ CTL responses through downregulation of peptide/MHC class I and Fas ligand-mediated cytotoxicity. J Immunol. 185(9): 5268-78.
[33] Segura E, Guérin C, Hogg N, Amigorena S, Théry C (2007) $\mathrm{CD}^{+}$dendritic cells use LFA-1 to capture MHC-peptide complexes from exosomes in vivo. J Immunol. 179(3): 1489-96.

[34] Morelli AE, Larregina AT, Shufesky WJ, Sullivan ML, Stolz DB, Papworth GD, Zahorchak AF, Logar AJ, Wang Z, Watkins SC, Falo LD Jr, Thomson AW (2004) Endocytosis, intracellular sorting, and processing of exosomes by dendritic cells. Blood. 104(10): 3257-66.

[35] Obregon C, Rothen-Rutishauser B, Gerber P, Gehr P, Nicod LP (2009) Active uptake of dendritic cellderived exovesicles by epithelial cells induces the release of inflammatory mediators through a TNFalpha-mediated pathway. Am J Pathol. 175(2): 696-705.

[36] Koumangoye RB, Sakwe AM, Goodwin JS, Patel T, Ochieng J (2011) Detachment of breast tumor cells induces rapid secretion of exosomes which subsequently mediate cellular adhesion and spreading. PLoS One. 6(9):e24234.

[37] Maecker HT, Todd SC, Levy S (1997) The tetraspanin superfamily: molecular facilitators. FASEB J. 11(6): 428-42.

[38] Zöller M (2009) Tetraspanins: push and pull in suppressing and promoting metastasis. Nat. Rev. Cancer. 9(1): 40-55.

[39] Hemler ME (2005) Tetraspanin functions and associated microdomains. Nat. Rev. Mol. Cell. Biol. 6(10): 801-11.

[40] Andre F, Escudier B, Angevin E, Tursz T, Zitvogel L (2004) Exosomes for cancer immunotherapy. Ann. Oncol. 15 Suppl 4: iv141-4.

[41] Piper RC, Katzmann DJ (2007) Biogenesis and function of multivesicular bodies. Annu. Rev. Cell Dev. Biol. 23: 519-47. 